\title{
Der Schwangerschaftsabbruch und sein Stellenwert in der medizinischen Versorgung
}

\author{
Die Initiative «Abtreibungsfinanzierung ist Privatsache» möchte die Kosten eines \\ Schwangerschaftsabbruchs aus dem Pflichtleistungs-Katalog der Grundversiche- \\ rung herausnehmen. Für Frauen mit knappen Ressourcen, Jugendliche und Migran- \\ tinnen könnte dies gesundheitlich schädliche, aber auch volkswirtschaftlich uner- \\ wünschte Wirkungen haben.
}

Die bevorstehende Volksabstimmung über die Abgeltung des Schwangerschaftsabbruchs (SA) durch die Krankenversicherung gibt die Gelegenheit, den Stellenwert des SA in der medizinischen Versorgung der Schweiz zu beleuchten. In der Schweiz ist etwa jede fünfte Frau mindestens einmal im Leben davon betroffen. Unerwünschte Schwangerschaften sind neben der individuellen existentiellen Bedrohlichkeit für Frauen im fruchtbaren Alter auch eine wichtige Grösse für die öffentliche Gesundheit. In den Ländern mit den geringsten Hindernissen für Frauen mit unerwünschten Schwangerschaften ist die Rate der SA besonders tief.

In der Schweiz werden jährlich rund 11000 SA vorgenommen [1]. Die Rate von 6,8 SA pro 1000 Frauen im gebärfähigen Alter ist die tiefste in Europa und möglicherweise weltweit. Die Zahl der SA ist bis in die 80er Jahre drastisch gesunken. In der vergangenen Dekade verminderte sie sich nur noch wenig. Die Geburten haben dagegen in den vergangenen Jahren wieder zugenommen. Im Jahr $2011 \mathrm{ka}-$ men 132 SA auf 1000 Lebendgeburten.

Der Zugang zum SA ist in der Schweiz für die meisten Frauen mit unerwünschter Schwangerschaft nicht schwierig und ist ohne Zeitverzug gewährleistet. Die meisten Schwangerschaftsabbrüche erfolgen im Zeitraum von weniger als 5 Wochen nach der Empfängnis ( $<7$ Wochen Amenorrhoe). Zwei Drittel der Abtreibungen werden mit Medikamenten alleine und nur ein Drittel mit chirurgischen Mitteln vorgenommen. Spätabbrüche sind selten: 2011 erfolgten nur 5\% nach der 12. Schwangerschaftswoche wegen besonders schwerwiegenden Gründen. Teenagerschwangerschaften sind in der Schweiz selten. Im Alter von 15 bis 19 Jahren hatten 4,6 Frauen oder Mädchen pro 1000 einen SA. 1\% der SA betrifft Mädchen von weniger als 16 Jahren.

Für Frauen im fruchtbaren Alter ist ungenügende medizinische Betreuung von Schwangerschaften weltweit nach wie vor die wichtigste Ursache von Krankheit und Tod. Jährlich sterben 47000 Frauen an den Folgen von nicht fachkundig durchgeführten SA [2]. Dieser Zusammenhang zeigt sich nicht nur in

\section{Quelle est la place de}

\section{I'interruption de grossesse dans}

\section{les soins médicaux en Suisse?}

L'initiative populaire fédérale «Financer l'avortement est une affaire privée» entend radier les coûts de l'interruption de grossesse (IVG) du catalogue des prestations prises en charge par l'assurance obligatoire de base. Or, cela pourrait avoir des conséquences néfastes non seulement sur la santé des femmes à faible revenu, des jeunes et des migrantes, mais aussi sur l'économie.

En Suisse, la prise en charge médicale des grossesses non désirées est excellente en comparaison internationale. Les consultations, les informations et les contraceptifs sont faciles d'accès pour la grande majorité des femmes et le nombre d'IVG y est plus bas que dans les autres pays à l'échelle européenne, voire mondiale: 11000 IVG par an, soit un taux de 6,8 IVG pour 1000 femmes en âge de procréer.

L'article ci-après traite de la prise en charge médicale des IVG, des lacunes à combler et du potentiel d'amélioration dans ce domaine.

wenig entwickelten Ländern: Zum Beispiel starben in Portugal bis zur Gesetzesreform im Jahr 2007 jährlich mehrere Frauen an den Folgen von Pfuschereien; 28\% der Frauen mussten wegen Komplikationen hospitalisiert werden [3]. In den meisten Ländern Westeuropas aber ist der Zugang zu Kontrazeptiva und medizinisch korrekt durchgeführten Schwangerschaftsabbrüchen gewährleistet. Schwere oder gar tödliche Komplikationen sind extrem selten. 


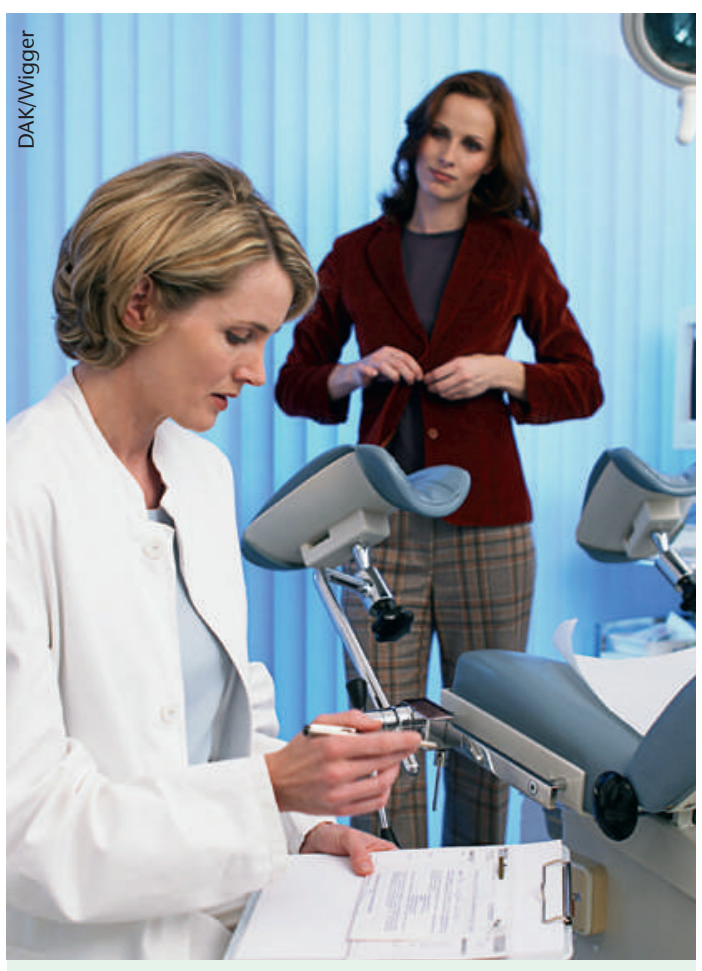

6,8 Schwangerschaftsabbrüche pro 1000 Frauen im gebärfähigen Alter: Diese Rate in der Schweiz ist die tiefste Europas.

In der Schweiz sind laut bundesamtlicher Statistik seit 1980 keine Todesfälle in Zusammenhang mit SA bekannt geworden, dagegen sterben 5,2 Frauen pro 100000 Geburten Grössere Untersuchungen in Grossbritannien [4] zeigen 2004 eine mütterliche Mortalität von 6 auf 100000 Geburten gegenüber 0,2 pro $100000 \mathrm{SA}$ und bestätigen die Zahl von zwei Todesfällen pro Million SA aus amerikanischen früheren Untersuchungen. Infektionen mit Clostridium sordellii haben in Amerika zu insgesamt einem knappen Dutzend Todesfällen beim medikamentösen SA geführt, in Europa ist nur ein einziger Fall bekannt geworden.

Auch in Bezug auf nicht-tödliche Komplikationen ist es für eine Frau um ein Vielfaches sicherer, ihre unerwünschte Schwangerschaft abzubrechen, als bis zur Geburt auszutragen und zu gebären [5]. Bei Abtreibungen können sehr selten Komplikationen der Anästhesie, schwere Blutungen oder schwere Infektionen beobachtet werden. Aufsteigende Infektionen sind meistens vorbestehend, und die Häufigkeit kann durch prophylaktische Antibiose (z. B. mit einem Gramm Azithromycin) kostengünstig reduziert werden. Komplikationshäufigkeiten korrelieren positiv mit der Schwangerschaftsdauer. Ein medikamentöser SA vor der 7. Schwangerschaftswoche ist das glimpflichste Vorgehen.

Perforationen der Uteruswand oder via falsa bei der Cervixdilatation sind beim chirurgischen SA gelegentlich [6] möglich, aber führen äusserst selten zu bleibenden Schäden. Bei Patientinnen mit endo- metrialen Synechien finden sich Abtreibungen in der Vorgeschichte deutlich gehäuft. Solche Verklebungen der Gebärmutterschleimhaut können beim chirurgischen SA durch möglichst schonendes Absaugen und sparsames Ausschaben mit dem scharfen Löffel anscheinend nicht immer vermieden werden.

Auch in grossen wissenschaftlichen Untersuchungen zeigt sich aber keine signifikant verminderte Fruchtbarkeit nach Abtreibungen [7]. Einflussgrössen (selection biases) wie beispielsweise die Häufigkeit von sexuell übertragbaren Infektionen, Suchtmittelkonsum, andere Risikoverhalten oder Migration verfälschen die Aussagekraft solcher Statistiken erheblich. Für die meisten Frauen ist die unerwünschte Schwangerschaft zunächst einmal der Beweis ihrer Fruchtbarkeit, und ein Gespräch über Kontrazeptiva ist vordringlich. Andere körperliche Folgen von SA werden immer wieder in Medien behauptet, aber entbehren der wissenschaftlichen Grundlagen.

Zweifellos ist eine unerwünschte Schwangerschaft im Leben einer Frau nicht selten ein markantes Ereignis und steht oft in Zusammenhang mit schwerwiegenden Krisen im unmittelbaren persönlichen Beziehungsfeld. Beziehungskrisen, unerwünschte Schwangerschaft und SA sind daher nicht überraschend oft prägende Inhalte von psychischen Störungen. Ein ursächlicher Zusammenhang von späteren psychischen Störungen kann aber in keinen grossen Studien nachgewiesen werden $[8,9]$. Frauen waren nach einem SA im ersten Trimester nicht öfter psychisch krank als vorher [10].

Die eidgenössische Initiative «Abtreibungsfinanzierung ist Privatsache» verlangt, dass die Kosten eines SA von der Grundversicherung der obligatorischen Krankenversicherung nicht mehr übernommen werden. Diese Änderungen von Art. 30 KVG \& Art. 119 StGB können für Frauen mit knappen Ressourcen, für Jugendliche und Migrantinnen gesundheitlich schädliche, aber auch volkswirtschaftlich unerwünschte Wirkungen haben. Amerikanische Untersuchungen [11] über die Auswirkungen der Streichung von Medicaid-Leistungen an den SA zeigen,

- dass die zusätzlichen öffentlichen Kosten den durch die Streichung der Finanzierung von Abtreibungen durch Medicaid eingesparten Betrag um ein 4- bis 5 faches übersteigen.

- dass sich der SA wegen Finanzierungsproblemen gefährlich verzögert.

- dass unerwünschte Schwangerschaften vermehrt ausgetragen werden.

- dass ein kleiner Prozentsatz von Frauen selber versucht, eine Abtreibung zu provozieren [12].

Die Kosten des SA belaufen sich gemäss einer Studie in der Romandie [13] auf durchschnittlich 1360 CHF, aber variierten erheblich von 400 CHF bis 3500 CHF. Der medikamentöse SA war mit durchschnittlich 1076 CHF billiger als das chirurgische Vorgehen mit 1490 CHF. Die Kosten sind nur teilweise abhän- 
gig von unterschiedlichem Aufwand. Die Tarifierung in der Grundversicherung ist auch ein Schutz vor hohen Preisen im privaten Bereich.

Die Kosten müssen von allen schweizerischen Krankenkassen in der Grundversicherung übernommen werden. Trotzdem werden SA oft nicht über die Kasse abgerechnet; in unserer eigenen Praxis bezahlt ein Drittel der Patientinnen selber. Gründe dafür sind ein hoher Franchisenbetrag oder der ungenügende Schutz ihrer Geheimsphäre bei Abrechnung durch die Krankenversicherung. Frauen müssen einen SA gegenüber ihren Eltern oder dem Partner oft verheimlichen. Zwang oder gar Gewalt bedroht nicht nur junge Frauen mit Migrationshintergrund. Frauen mit unerwünschter Schwangerschaft unterliegen in dieser Situation oft Pressionen, die ihre ganze Zukunft und unmittelbar ihre Gesundheit gefährden können.

Die medizinische Versorgung von Frauen mit unerwünschter Schwangerschaft weist in der Schweiz geringe Lücken auf. Seit der Volksabstimmung zur Revision der schweizerischen Strafgesetzbuchartikel 119 \& 120 («Fristenregelung») im Jahr 2002 sind die regionalen Unterschiede zurückgegangen. Nicht überall kann der SA ambulant oder medikamentös durchgeführt werden. Von 113 Schweizer Kliniken, die auf eine repräsentative Umfrage der APAC-Suisse [14] geantwortet haben, führen 93 (82\%) SA durch. Von diesen nehmen 69\% auch nach der 12 . Schwangerschaftswoche Abbrüche vor, aber bloss 49\% auch bei psychosozialer Indikation («schwere seelische Notlage», gemäss StGB Art. 119 Abs. 1).

Wenn der SA nach der 12. Schwangerschaftswoche in einem Kanton abgelehnt wird, kann die Frau aus versicherungstechnischen oder administrativen Gründen von den meisten Kliniken in anderen Kantonen nicht aufgenommen werden. Jährlich reisen rund 50 Frauen aus der Schweiz für einen SA nach dem ersten Trimenon ins Ausland.

\section{«Die volkswirtschaftlichen Gesamtkosten würden} voraussichtlich steigen.»

$\mathrm{Zu}$ unerwünschten Schwangerschaften führende Situationen sind oft mit einem erhöhten Risiko für sexuell übertragbare Infektionen (STI) verknüpft. Bei allen Schwangeren wäre eigentlich eine vollständige STI-Abklärung inklusive HIV-Screening angebracht. In unserer eigenen Praxis wurden bei den für einen SA voruntersuchten Frauen in 9\% Chlamydia trachomatis [15] und 1\% Neisseria Gonorrhoeae gefunden. Gerade bei besonders vulnerablen Frauen konnten weitere STI-Abklärungen oft nicht durchgeführt werden, unter anderem wegen Finanzierungsschwierigkeiten.
Eine allfällige Annahme der Initiative «Abtreibungsfinanzierung ist Privatsache» hätte negative öffentliche Auswirkungen. Die volkswirtschaftlichen Gesamtkosten würden voraussichtlich steigen und die reproduktive Gesundheit der Schweizer Frauen könnte beeinträchtigt werden. Erschwernisse im Zugang zum SA beispielsweise durch Finanzierungsprobleme treffen vor allem vulnerable Gruppen. Die Versorgung kann in der Schweiz vor allem für junge Frauen und Frauen mit Migrationshintergrund verbessert werden. Verbesserungen sehen wir vor allem in den Bereichen der Finanzierung von Kontrazeptiva und von Abklärungen bezügich STI.

\section{Literatur}

1 Bundesamt für Statistik: Schwangerschaftsabbrüche in der Schweiz, 2011. www.bfs.admin.ch/bfs/portal/de/ index/themen/14/02/03/key/03.html

2 Zusammenfassung auf der Website des Guttmacher Institute: www.guttmacher.org/media/evidencecheck/ index.html

3 Duarte Vilar: The Portuguese Experience. FIAPAC conference. Berlin; 2008.

4 RCOG: Saving Mothers' Lives: Reviewing maternal deaths to make motherhood safer: 2006-2008. The Eighth Report of the Confidential. Enquiries into Maternal Deaths in the UK. March 2011; Volume 118, Issue Suppl s1, 1-203

5 Rowlands S. Misinformation on abortion. The European Journal of Contraception and Reproductive Health Care. 2011; Early Online, 1-8.

6 Soulat C, Gelly M. Complications immédiates de l'IVG chirurgicale. J Gynecol Obstet Biol Reprod 2006;35:157-62.

7 Rowlands S. Misinformation on abortion. The European Journal of Contraception and Reproductive Health Care. 2011; Early Online, 1-8.

8 APA Task Force. Mental health and abortion. Washington, DC: American Psychological Association 2008

9 Charles VE, et al. Abortion and long-term mental health outcomes: A systematic review of the evidence. Contraception 2008;78:436-50.

10 Munk-Olsen T, et al. Induced First-Trimester Abortion and Risk of Mental Disorder. N Engl J Med. 2011;364:332-9.

11 Henshaw SK, et al. Restrictions on Medicaid Funding for Abortions: A Literature Review. July 2009. www. guttmacher.org/pubs/MedicaidLitReview.pdf

12 Reproductive Health Matters, Volume 18, Issue 36 , Seiten 136-146, November 2010.

13 Perrin E, et al. Clinical course in women undergoing termination of pregnancy within the legal time limit in French speaking Switzerland. Swiss Med Wkly. 2011;141:w13282.

14 Rey AM, Seidenberg A. Schwangerschaftsabbruch: die Praxis der Spitäler und Kliniken in der Schweiz. Die Ergebnisse einer Umfrage der APAC-Suisse aus dem Jahr 2009. Schweiz Ärztezeitung. 2010;91(13/14):551-4.

15 Wesbonk JP. Prevalence and determinants of sexually transmitted infections in women undergoing abortion in a Swiss primary care setting. Diss. Universität Zürich 2013, eingereicht. 